\title{
Combining Ablation and Resection for the Treatment of Hepatocellular Carcinoma: An Attempt to Expand Treatment Options
}

\author{
Essa M. Aleassa, $\mathrm{MD}^{1,2}$, and Koji Hashimoto, $\mathrm{MD}, \mathrm{PhD}^{1}$ \\ ${ }^{1}$ Department of General Surgery, Digestive Disease and Surgery Institute, Cleveland Clinic, Cleveland, OH; ${ }^{2}$ Department \\ of Surgery, College of Medicine and Health Sciences, United Arab Emirates University, Al Ain, Abu Dhabi, United Arab \\ Emirates
}

Despite the potential eradication of viral hepatitis B and $\mathrm{C}$, the incidence of hepatocellular carcinoma (HCC) is increasing due to the rising prevalence of obesity and nonalcoholic steatohepatitis. ${ }^{1,2}$ Surgical resection is one of the established treatments for select patients with HCC. However, safe resection is precluded and necessary extensive resection leaves patients with suboptimal liver remnant in circumstances where the liver lesions are either multifocal, located deep in the liver, or abutting vital structures. $^{3}$ On the other hand, liver transplantation simultaneously offers oncologically better local control with total hepatectomy and treatment of background liver disease. Although an increasing number of patients have received liver transplantation with expanded morphological criteria, ${ }^{4,5}$ the ever-short supply of donor organs renders many transplant candidates remaining on the waitlist until the disease progresses or the overall condition deteriorates. Therefore, it is essential to come up with alternative strategies to treat HCC.

Combining liver resection with alternative local therapies such as tumor ablation, e.g. radiofrequency ablation (RFA), has been described for HCC as a solution to the dilemma described above. The study by Huang et al. ${ }^{6}$ in the current issue compares their single-institution, short- and long-term outcomes of applying either resection only

(C) Society of Surgical Oncology 2020

First Received: 9 January 2020;

Published Online: 27 January 2020

K. Hashimoto, MD, PhD

e-mail: HASHIMK@ccf.org versus a combined approach in multifocal HCC patients. The study included 210 and 51 patients in the resection alone and combined approach cohorts, respectively. The authors also analyzed the results after applying a propensity score-matched analysis, which dropped the sample size to 43 patients in each group.

The authors report similar overall survival rates at 1, 3, and 5 years between the two groups (combined approach: $86 \%, 64.7 \%$, and $39.7 \%$ versus resection only: $90.6 \%$, $67.4 \%$, and $42.7 \% ; p=0.592$ ). Similar results were also noted regarding recurrence-free survival rates at 1,3 , and 5 years (combined approach: $83.7 \%, 38.3 \%$, and $22.7 \%$ versus resection only: $81.4 \%, 55.9 \%$, and $29.7 \%$; $p=0.361$ ). A subgroup analysis demonstrated that patients with two tumors had superior recurrence-free survival, at 1 , 3 , and 5 years if they underwent liver resection alone $(88.7 \%, 59.9 \%$, and $30.1 \%$, respectively) as opposed to combined resection and RFA $(81.8 \%, 40 \%$, and $22.9 \%$, respectively; $p=0.025)$. However, overall survival did not differ between the treatment groups. Patients with three tumors did not differ in overall survival or recurrence rates. When assessing the effect of tumor size, hepatic resection alone yielded better recurrence-free survival than the combined approach in patients with a dominant mass of $\leq 3 \mathrm{~cm}(86.8 \%, 56.8 \%$, and $27.6 \%$ versus $82.6 \%, 37.9 \%$, and $15.2 \%$, respectively; $p=0.048$ ).

The study in hand is the largest study reporting outcomes of such interventions in primary HCC patients. Previously, a meta-analysis by $\mathrm{Xu}$ et al., including 466 patients from four retrospective studies, compared survival outcomes between the combined approach $(n=197)$ and liver resection only $(n=269)$ in patients with primary HCC. The pooled results showed that at 3 years, the overall 
and disease-free survival were comparable between the two groups. ${ }^{7}$ A similar retrospective study by Hiraoka et al. reported the results of 82 patients receiving resection only and 33 patients using the combined approach, but RFA was percutaneously performed separately from liver resection. Patients in this cohort did not necessarily satisfy the Milan or University of California San Francisco (UCSF) criteria. Again, there was no significant difference in the 5 -year overall survival $(65.6 \%$ and $67 \% ; p=0.244)$ or disease-free survival $(23.3 \%$ and $28 \% ; p=0.257)$ between the two treatments, respectively. ${ }^{8}$

It is promising to see that both approaches are comparable in overall and disease-free survival; however, a few studies reported recurrence at the site of ablation, which was not stated in the current study. The recurrence rate noted in the literature regarding the RFA sites was in the range of $16.7-20.5 \%$, which is higher than to be neglected. ${ }^{9,10}$ This might make the combined approach a secondline therapy, given the proven non-inferiority of the procedure as opposed to the primary option.

The inherit risk of HCC recurrence after ablation needs to be factored into the management decision if resection of all lesions is a viable option. Several factors have been identified as culprits for local recurrences, such as incomplete ablation due to technical error or misjudgment, aggressive tumor biology, heat-sink effect, and underlying liver disease. ${ }^{11}$ However, when lesions are not amenable for resection without sacrificing a significant liver volume, the combined approach would be a better option to achieve long-term survival. The other patient cohort suitable for the combined approach is those with cirrhosis demanding a higher remnant liver volume. However, it should be noted that portal hypertension is one of the unfavorable factors for long-term survival.

The study by Huang et al. should be applied to local practices cautiously as it might incur selection bias. They were very cautious with proceeding with surgery by excluding patients with generally acceptable remnant liver volumes, while other institutes would have proceeded with surgery (remnant volume $<40 \%$ in non-cirrhotic patients and $<50 \%$ in cirrhotic patients). ${ }^{12}$ Furthermore, the study is missing data regarding the types of liver resections and remnant liver volumes, which would influence perioperative outcomes. Nonetheless, the combined approach allows patients who are deemed unsuitable for resection alone to undergo curative treatment. Prospective multicenter studies are needed to better characterize the patient population who achieve long-term survival with the combined approach.
DISCLOSURE Essa M. Aleassa and Koji Hashimoto declare no conflicts of interest.

\section{REFERENCES}

1. Baecker A, Liu X, La Vecchia C, Zhang ZF. Worldwide incidence of hepatocellular carcinoma cases attributable to major risk factors. Eur J Cancer Prev. 2018;27(3):205-12.

2. Younossi Z, Anstee QM, Marietti M, et al. Global burden of NAFLD and NASH: trends, predictions, risk factors and prevention. Nat Rev Gastroenterol Hepatol. 2018;15(1):11-20.

3. Kow AWC. Transplantation versus liver resection in patients with hepatocellular carcinoma. Transl Gastroenterol Hepatol. 2019;4:33.

4. Mazzaferro V, Regalia E, Doci R, et al. Liver transplantation for the treatment of small hepatocellular carcinomas in patients with cirrhosis. N Engl J Med. 1996;334(11):693-99.

5. Yao FY, Ferrell L, Bass NM, et al. Liver transplantation for hepatocellular carcinoma: expansion of the tumor size limits does not adversely impact survival. Hepatology. 2001;33(6):1394-1403.

6. Huang Y, Song J, Zheng J, et al. Comparison of hepatic resection combined with intraoperative radiofrequency ablation, or hepatic resection alone, for hepatocellular carcinoma patients with multifocal tumors meeting the University of California San Francisco (UCSF) criteria: a propensity score-matched analysis. Ann Surg Oncol. 2020. https://doi.org/10.1245/s10434-020-08231-0.

7. Xu LL, Zhang M, Yi PS, et al. Hepatic resection combined with radiofrequency ablation versus hepatic resection alone for multifocal hepatocellular carcinomas: a meta-analysis. J Huazhong Univ Sci Technol Med Sci. 2017;37(6):974-80.

8. Hiraoka A, Hirooka M, Ochi H, et al. Combination of resection and ablative treatment for hepatocellular carcinoma: usefulness of complementary radiofrequency ablation. Oncology. 2019;96(5):242-51.

9. Cheung TT, Ng KK, Chok KS, et al. Combined resection and radiofrequency ablation for multifocal hepatocellular carcinoma: prognosis and outcomes. World $J$ Gastroenterol. 2010;16(24):3056-62.

10. Lee SJ, Cho EH, Kim R, Kim YH, Lim CS, Kim SB. Hepatectomy, combined with intraoperative radiofrequency ablation in patients with multiple hepatocellular carcinomas. Korean $J$ Hepatobiliary Pancreat Surg. 2015;19(3):98-102.

11. Ganne-Carrie N, Nault JC, Ziol M, et al. Predicting recurrence following radiofrequency percutaneous ablation for hepatocellular carcinoma. Hepat Oncol. 2014;1(4):395-408.

12. Yigitler C, Farges O, Kianmanesh R, Regimbeau JM, Abdalla EK, Belghiti J. The small remnant liver after major liver resection: how common and how relevant? Liver Transplant. 2003;9(9):S18-25.

Publisher's Note Springer Nature remains neutral with regard to jurisdictional claims in published maps and institutional affiliations. 